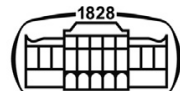

AKADÉMIAI KIADÓ

UNIVERSITY of DEBRECEN

\section{International Review of Applied Sciences and Engineering}

$12(2021) 3,312-323$

DOI:

10.1556/1848.2021.00306

(c) 2021 The Author(s)

\title{
A statistics-based review of microgrid protection with a concentration on adaptive protection
}

\author{
Amin Damanjani, Mohamad Hosseini Abardeh* ${ }^{\circ}$, \\ Azita Azarfar and Mehrdad Hojjat \\ Department of Electrical and Computer Engineering, Shahrood Branch, Islamic Azad University, \\ Shahrood, Iran
}

Received: April 30, 2021 • Accepted: June 9, 2021

Published online: June 26, 2021

\section{ABSTRACT}

Microgrid (MG) is a system of production and distribution of electrical energy that can operate both in grid-connected and islanded modes. This capability leads to significant variations in the fault current level. Moreover, dynamic changes corresponding to the line outage contingencies or outages of the distributed generations (DGs) that are implemented for local generation in the MGs lead to the changes in the fault current level. These changes in the fault current level make some miscoordination between the overcurrent relays (OCRs) in the conventional protection schemes. To overcome this drawback, there is a need for an adaptive protection scheme that can adapt to both operational and dynamic changes and takes effective protection decisions accordingly. This paper first presents a statistic-based review of MG and its protection including total publications, type of publications, the ten most researchers, and the ten most sources. Finally, comprehensive remarks of the 30 most cited papers related to adaptive protection of MG are presented. This paper will help the researchers of the MG protection to learn the most desirable techniques and the concentration of studies in adaptive protection of MGs for future works.

\section{KEYWORDS}

microgrid, adaptive protection, statistics, overcurrent relays, power system protection

\section{INTRODUCTION}

Nowadays, decentralization of power systems is possible utilizing distributed generators (DGs) in the microgrids (MGs) to feed the local loads or inject excess power to the main grid. MG is a section of the power system facilitating the integration of DGs, electric storage systems (ESSs), and loads [1]. Also, MGs are capable of operating in both islanded and gridconnected modes [2].

As illustrated in Fig. 1, the energy sources in MGs consist of wind turbines (WTs), photovoltaic (PV) units, and fuel cells (FCs) [3]. Moreover, the ESSs, in which battery energy storage (BES) is considered as the best option among them, are reliable devices to mitigate the volatility and intermittency of renewable DGs and are utilized in MGs [4].

The major advantages of utilizing MGs in the power system can be stated as follows [5-7]:

- Capability to operate in two modes of operation;

- Capability to integrate a wide variety of power resources;

- Improvement of local economic growth and jobs;

*Corresponding author.

E-mail: mohamad.hosseini@mail.um. ac.ir
- Being a source of income by injecting the generated power to the utility grid;

- Ability to handle the peak loads by feeding the loads in MGs in islanded mode or injecting the excess generation to the main grid;

- Enhancing the cyber-security;

- High efficiency due to generation near load

- Reduction in investment costs in comparison to large power plants; 


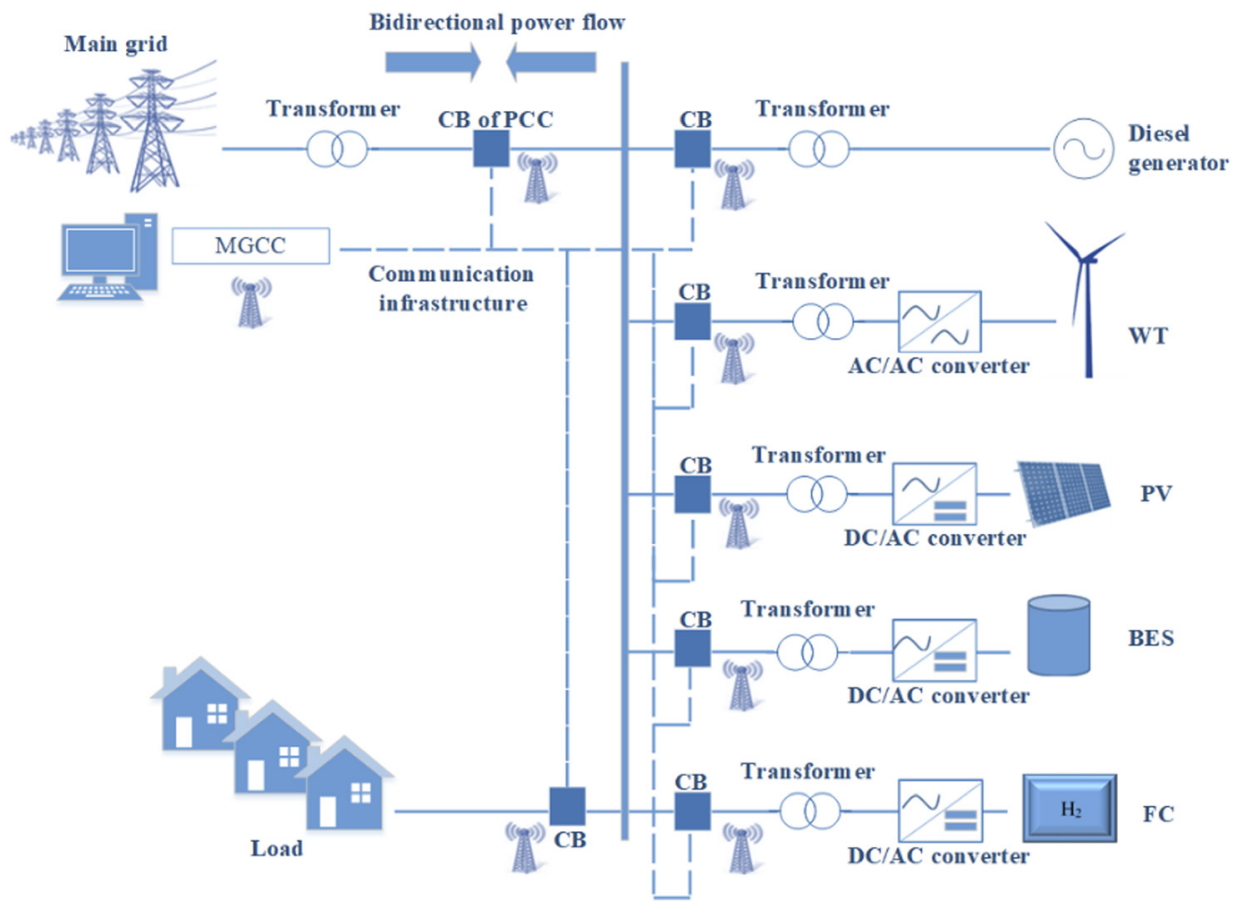

Fig. 1. Structure of a MG

- Reduction of the environmental impacts;

- Capability to manage and control the uninterrupted energy production;

- Capability of managing the fluctuations caused by the load demand;

- Reduction of the transmission losses in the system;

- Improving the power reliability due to the ability to work in two operation modes;

- Quicker installation in comparison to the traditional power plant.

Also, a MG consists of communication infrastructure for control and protection purposes. The communication infrastructures can be divided into wired and wireless implementations [8].

In wired communication-based infrastructures, the data transfer between intelligent electronic devices (IEDs) and the MG central controller (MGCC) happens through the cables [8].

On the other hand, wireless communication is based on communication protocols including IEC 61850, the distributed network protocol 3.0 (DNP 3.0), the process field bus (Profibus), the Modbus, Wi-Fi, and the transmission control protocol/internet protocol (TCP/IP) [9]. The schematic diagram of communication infrastructure (both wired and wireless infrastructures) is shown in Fig. 1.

It is worth noting that the MGCC is utilized in MGs for collecting and processing information and the system will collapse in the situation when MGCC fails [10]. MGCC with its connection to the MG's equipment is shown in Fig. 1.

In MG, the overcurrent relays (OCRs) encounter significant variation in fault currents due to the transition between the operation modes. A circuit breaker (CB) at the point of common coupling (PCC) is responsible for the transition between the two operation modes in MG. The CB of PCC isolates the MG from the main grid. Changes in MG's operation mode due to maintenance operation or load managing processes may lead to a change in the fault current level. In grid-connected mode, the network is fed by the main grid while in islanded mode, the fault current level seen by OCRs is only supplied by the sources that exist in the MG that leads to a reduction in the fault current level [10]. This reduction in fault current level in islanded mode leads to miscoordination in OCRs. Also, the ability of MG in working at two different operation modes encounters the OCRs to bidirectional power flow that leads to protection problems and the necessity of employing the directional OCRs. Moreover, the dynamic changes as the outage of MG's elements such as DGs or lines make changes of fault current level that leads to miscoordination between the OCRs. Consequently, utilizing the conventional protection schemes may lead to failure in the OCRs operation.

So, there is a need for a scheme to handle these situations and adapt the MG to the new situation and prevent protection problems. Numerous researches have been performed to overcome these protection problems in MGs. Adaptive protection is one of the most effective strategies to resolve these issues.

This paper aims to present a comprehensive analysis of the notable publications in MGs and especially adaptive protection issues. This will help the MG researchers to concentrate on the most desirable issues for future works.

The paper is organized as follows: Section 2 addresses the procedure of search. Section 3 gives statistics corresponding to $\mathrm{MG}$ and its protection and introduces the challenges in 
MG's protection. Section 4 introduces the adaptive protection in MG, presents the statistics on this issue, and addresses the most cited researches of adaptive protection in MG in detail. Finally, Section 5 gives the conclusions.

\section{PROCEDURE OF SEARCH}

In this paper, important information about publications in MG, its protection, and adaptive protection is highlighted, and statistical analysis on these topics are presented. The search procedure is carried out based on using widely popular databases, which include DOAJ, Nature Index journals, UGC Journal List, ERA, PubMed, J-STAGE, ERIH PLUS, and SciELO. For all statistics presented in this paper on any special search keyword, the total publications along with the separate number of publications by year from 2012 to 2021 (the statistics for 2021 include the first three months), the type of publications (i.e., journal papers, conference papers, or other publications including books, edited books, chapters, and monographs), the ten most researchers, and the ten most sources are presented. For the sake of clarity, a framework of the research procedure for this review paper is presented in Fig. 2. This information can help the researchers to learn the scope of concentration.

The keywords are chosen by two, three (or more) words separated by the term "and". Moreover, to make sure that the search is exactly on a special keyword, the search is limited to the title and abstract of the databases.

\section{MICROGRID PROTECTION AND CHALLENGES}

Protection is an important and desirable issue among the researchers of MGs. To make a comparison of how many studies are on MG and how many of them address the MG's protection, first, there is a statistical search available by

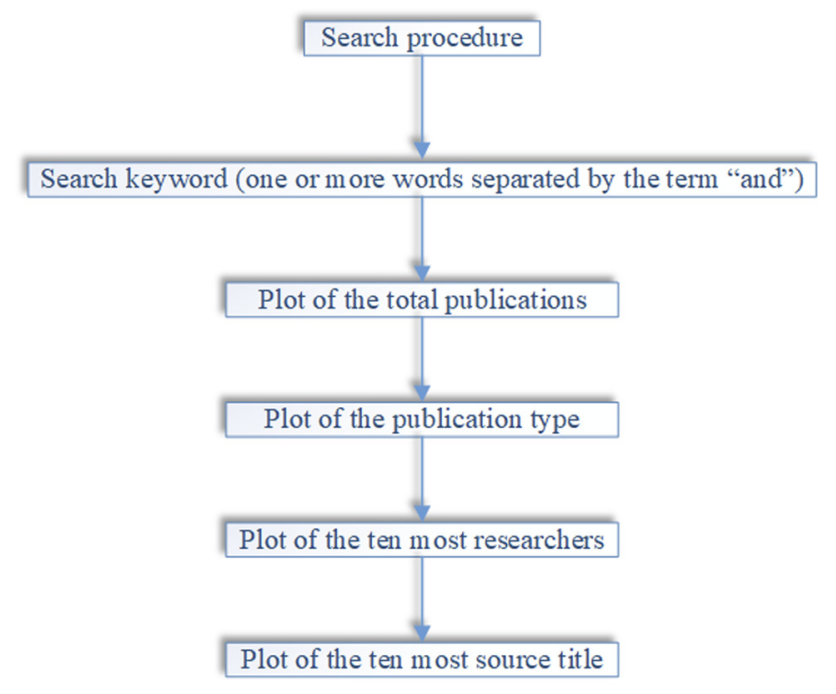

Fig. 2. A framework of the search procedure in this review paper keyword "Microgrid" in Fig. 3. The total number of publications, the type of publications, the ten most researchers, and the ten most publications of MG's issues are shown in Figs 3(a)-(d), respectively.

As can be seen from Fig. 3, most publications corresponded to the year 2020. Also, "J. M. Guerrero" with 650 publications on MG's issue has played a vital role in MG's research. Moreover, for the keyword "Microgrid and Protection", the statistical analysis is available in Fig. 4. As can be seen from Fig. 4(b), 47 percent of the researches are published in journals. Also, according to Fig. 4(c), "O. A. Mohammed" and "J. M. Guerrero" each with 30 publications are on the top of the researchers' list.

As illustrated in Figs 3(a) and 4(a) the total publications is 25,521 for the "Microgrid" topic and 1,417 for the "Microgrid and Protection" issue. It can be concluded that 18 percent of issues on MG have corresponded to its protection problems. This illustrates that a significant portion of MG publications is concentrated on protection issues. There are several challenges that should be taken into consideration in designing a proper protection scheme for a MG. The following subsections discuss the two major challenges in the protection of a MG.

\subsection{Challenges due to operational changes in $M G$}

Fault current level is changed significantly in transition between the grid-connected mode to islanded mode. In other words, in grid-connected mode, fault current includes a much higher magnitude in comparison to islanded mode. This reduction in fault current level in islanded mode causes malfunction of OCRs [11].

\subsection{Challenges due to dynamic changes in MG}

The dynamic changes in MG should be taken into consideration in designing a protection scheme. One of the main dynamic changes in MGs is the DGs' status (connected or disconnected). These two statuses of DGs may happen unintentionally due to fault occurrence or intentionally due to load managing. Different statuses of DGs' connections change in the fault current level and affect the operation of the OCRs' settings [12]. Moreover, line outage contingencies as dynamic changes lead to changes in fault current level. Issue of line outage in the power system can occur both planned such as network maintenance operations and unplanned such as fault situations. These issues may cause some problems for the protection system. In other words, the changes of fault current due to line outage contingencies must be considered in the protection scheme to prevent miscoordination of OCRs. So, an adaptive protection scheme is required to change the OCRs' settings according to the abovementioned challenges in MG.

\section{ADAPTIVE PROTECTION IN MG}

Adaptive protection is an online process that modifies the preferred protective responses to a change in system 

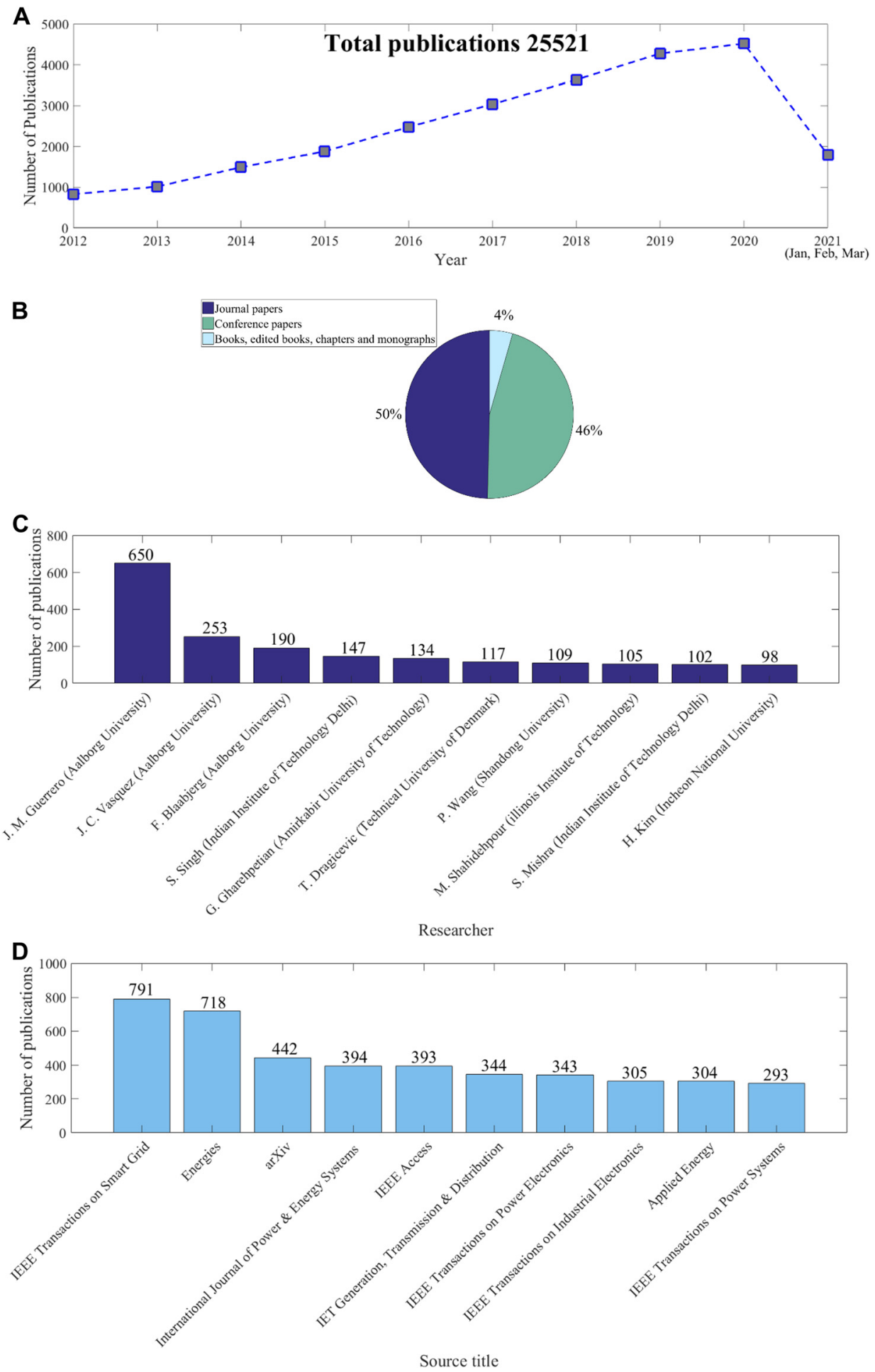

Fig. 3. Keyword "Microgrid" (a) Number of publications (b) Publication type (c) Ten most researchers (d) Ten most source title

condition [13]. Adaptive protection includes monitoring the MG parameters, detect the faults and perform adaptive protection actions accordingly.

A collection of publications details on adaptive protection in MG have been presented in Fig. 5. Figure 5(a) presents the annual number of publications by the term "Microgrid and Adaptive Protection". Moreover, Figs 5(b)(d) present the publications type, the ten most researchers, and the ten most journals, respectively, that work on this issue. As shown in Fig. 5(a), it is obtained there was a significant increase in the number of publications for adaptive protection in MGs in 2019 that shows there is still a significant need for more works on adaptive protection in MGs. Also, from Fig. 5(b) it is obtained that 51 percent of publications on adaptive protection in MGs are journal papers. Moreover, it is obtained from Fig. 5(c) that 
A
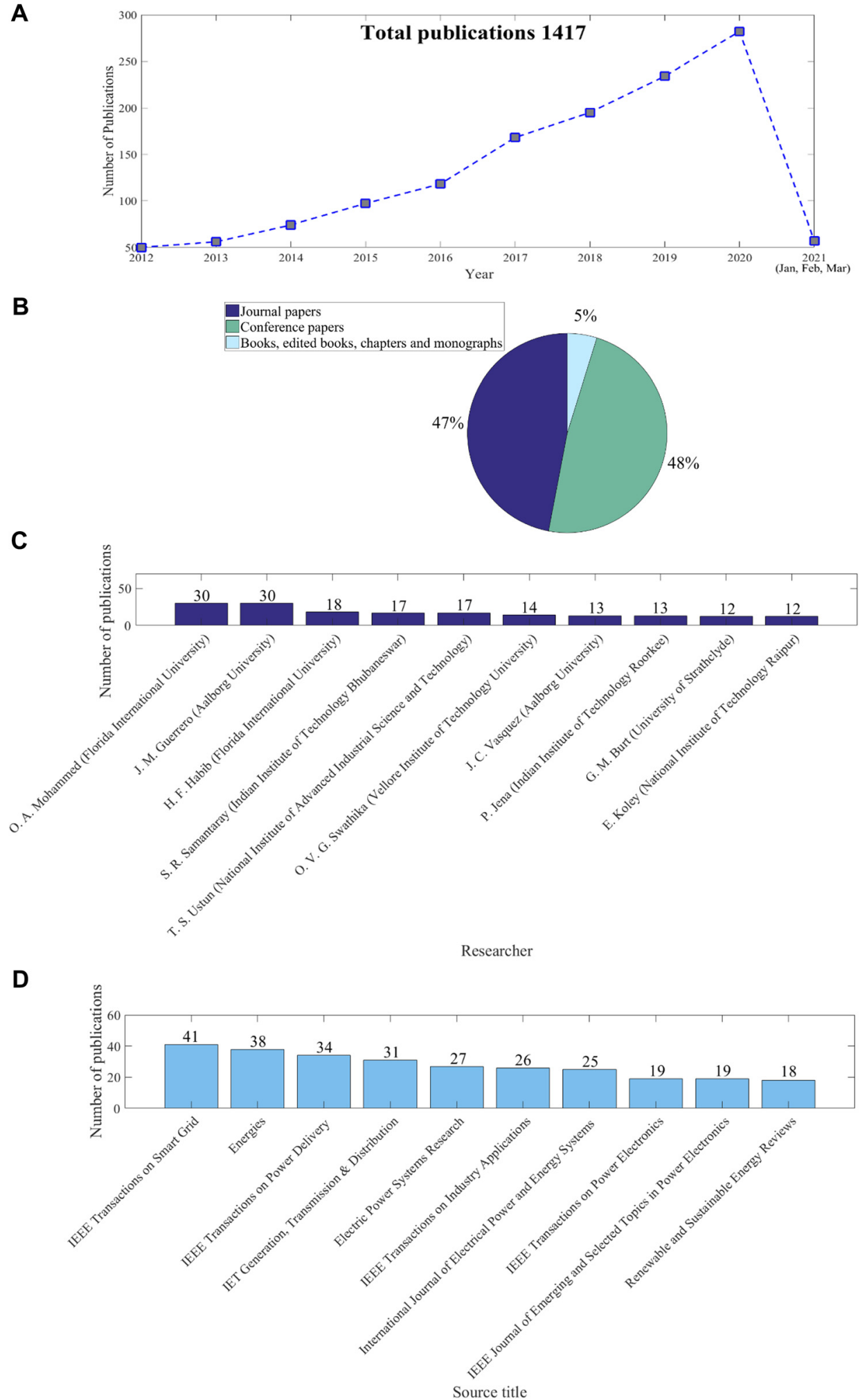

Fig. 4. Keyword "Microgrid and Protection" (a) Number of publications (b) Publication type (c) Ten most researchers (d) Ten most source title

"O. A. Mohammed" is on the top of the researchers' list. Besides, Fig. 5(d) illustrated that the journal "IET Generation, Transmission \& Distribution" has published the most papers on the issue of microgrid and adaptive protection.
To investigate the details of adaptive protection for MGs, first, the existing techniques are categorized. This will help the researchers to know which category is on the scope of the researchers' field of study. Then, the 30 most cited 
A

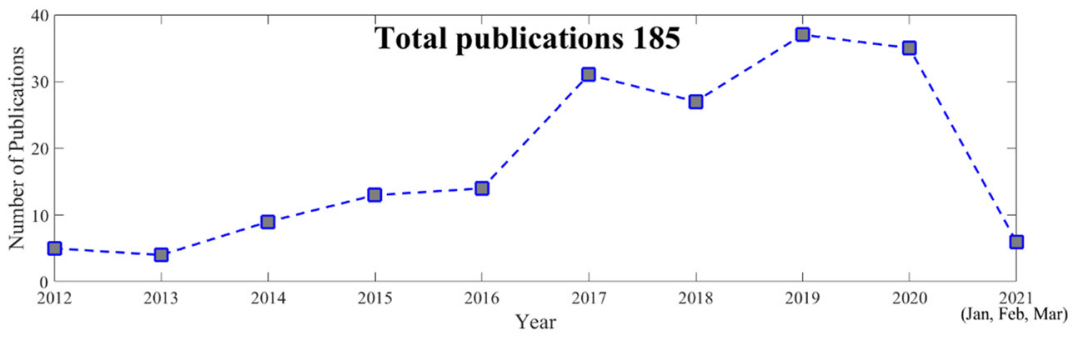

B
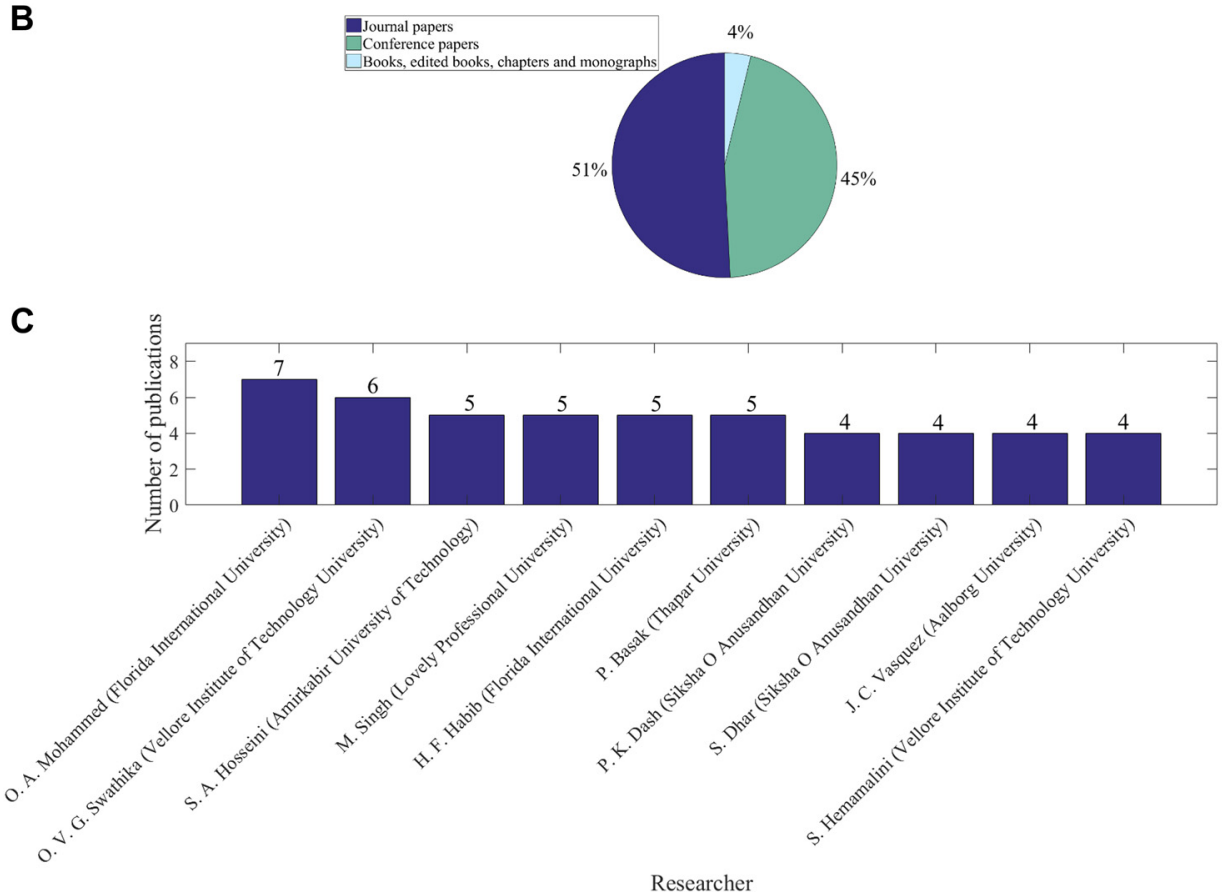

D

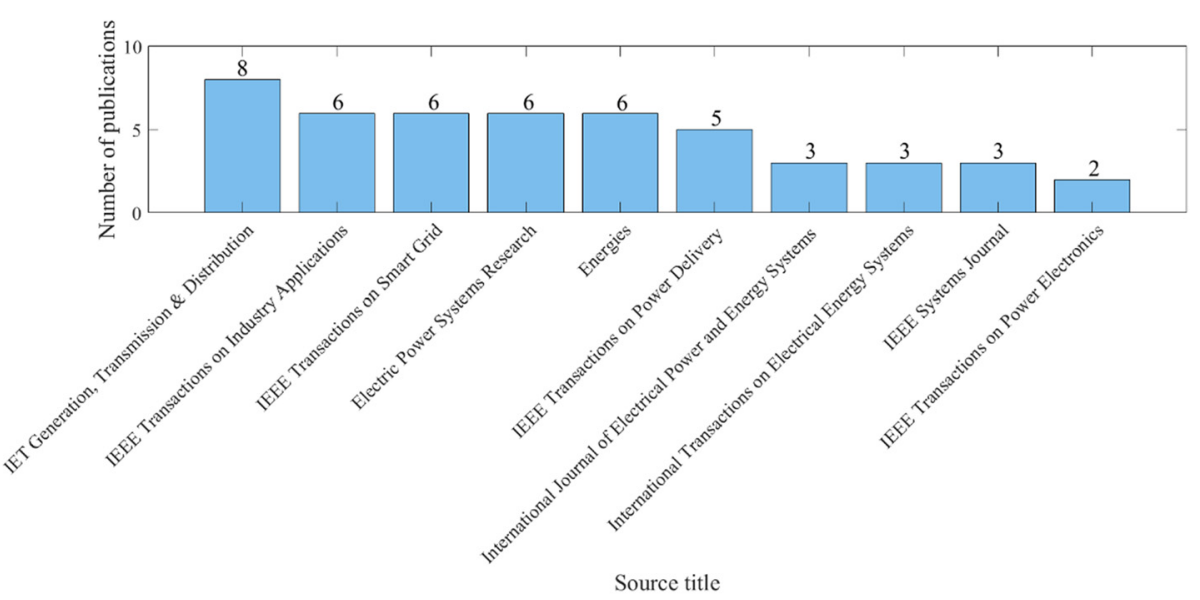

Fig. 5. Keyword "Microgrid and Adaptive Protection" (a) Number of publications (b) Publication type (c) Ten most researchers (d) Ten most source title

papers are investigated by details and labeled by the predefined categorizations. Moreover, the advantages of each research are remarked.

Generally, the existing techniques for adaptive protection of MG can be divided into centralized and decentralized categories.

Centralized architecture is supported by various communication protocols to transfer data to the MGCC for subsequent actions. On the other hand, the decentralized control architecture depends on the relationship between IEDs that are responsible for sending and receiving data. Each IED receives data from other IEDs and changes data (i.e., OCRs' settings) accordingly.

So, by the abovementioned definition for categorization related to MG's adaptive protection, there is a comprehensive search on the 30 most cited publications on this issue. Moreover, the information of references rank of citation, and a brief of remarks and advantages are summarized in Table 1. 
Table 1. The 30 most cited publications related to adaptive protection of MG (publications with a search of the term "Microgrid and Adaptive Protection")

\begin{tabular}{|c|c|c|c|c|}
\hline Reference & Citations & Rank & $\begin{array}{c}\text { Category (Centralized or } \\
\text { decentralized) }\end{array}$ & Remarks/advantages \\
\hline [14] & 212 & 1 & Decentralized & $\begin{array}{l}\text { Utilized the programmable microprocessor-based OCRs } \\
\text { Does not require communications infrastructure } \\
\text { Being independent of the fault current magnitude } \\
\\
\text { Being independent of the operation mode }\end{array}$ \\
\hline [15] & 123 & 2 & $\#$ & $\begin{array}{l}\text { - Presented a detailed review of protection schemes (both adaptive } \\
\text { and non-adaptive schemes) } \\
\text { - Dealt with schemes focused on fault currents and the changes in } \\
\text { the network topologies }\end{array}$ \\
\hline [16] & 108 & 3 & Decentralized & $\begin{array}{l}\text { - Utilized the communication-assisted OCRs that provided sufficient } \\
\text { fault current in fault situation for OCRs by the synchronous } \\
\text { generator in the MG } \\
\text { fapable to adapt the settings to OCRs dependent on the higher } \\
\text { fault current level in grid-connected mode and lower fault current } \\
\text { level in islanded mode }\end{array}$ \\
\hline [17] & 94 & 4 & Centralized & $\begin{array}{l}\text { - A centralized adaptive protection scheme to select the settings for } \\
\text { OCRs utilizing programmed logic } \\
\\
\text { utilizing IEC } 61850\end{array}$ \\
\hline [18] & 83 & 5 & Centralized & $\begin{array}{l}\text { - Monitored the MG and updated the OCRs' fault current according } \\
\text { to the fault } \\
\text { Utilized auto reclosers for a faster recovery to improve the } \\
\text { stability of the MG }\end{array}$ \\
\hline [19] & 80 & 6 & \# & $\begin{array}{l}\text { - Briefly reviews protection issues in MGs } \\
\text { - Investigated the limitations of protection schemes and improved } \\
\text { the reliability in communication infrastructures for adaptive pro- } \\
\text { tection schemes by alternative techniques }\end{array}$ \\
\hline [20] & 76 & 7 & $\#$ & $\begin{array}{l}\text { - Presented a summary of studies in the field of MG protection } \\
\text { - Analyzed the innovative methods proposed to solve protection } \\
\text { problems such as changing the OCRs' settings, the connection } \\
\text { status of DGs including the different types of DGs, the application } \\
\text { of FCLs, and adaptive protection }\end{array}$ \\
\hline [21] & 64 & 8 & Centralized & $\begin{array}{l}\text { - Utilized ESDs to increase the fault current magnitude in failure of } \\
\text { communications in the islanded mode } \\
\checkmark \text { Enhanced flexibility of MG against communication failures }\end{array}$ \\
\hline [22] & 55 & 9 & Centralized & $\begin{array}{l}\text { - Introduced a hybrid differential and adaptive scheme for MG's } \\
\text { protection utilizing a central controller } \\
\\
\text { Increased the accuracy and precision of the protection scheme } \\
\text { Reduced the overall communications cost }\end{array}$ \\
\hline [23] & 53 & 10 & Centralized & $\begin{array}{l}\text { - Obtained the adaptive fault current by considering the PSQ and } \\
\text { NSQ of fault currents } \\
\text { - Detected the fault by PSQ and NSQ of fault currents and adapted } \\
\text { the corresponded settings to the OCRs } \\
\\
\text { tions } \\
\\
\text { Improvided satisfactory protection coordination for fault situa- } \\
\end{array}$ \\
\hline [24] & 45 & 11 & Centralized & $\begin{array}{l}\text { - Used the negative sequence and the zero sequence of fault current } \\
\text { for fault detection } \\
\text { - Extracted the component of fault current by Fourier filter algo- } \\
\text { rithm } \\
\text { - Protected a MG in both the grid-connected and the islanded modes } \\
\text { by comparing the system impedance and MG side impedance } \\
\\
\text { Detected both operational and topological changes }\end{array}$ \\
\hline
\end{tabular}


Table 1. Continued

\begin{tabular}{|c|c|c|c|c|}
\hline Reference & Citations & Rank & $\begin{array}{c}\text { Category (Centralized or } \\
\text { decentralized) }\end{array}$ & Remarks/advantages \\
\hline$[25]$ & 43 & 12 & Decentralized & $\begin{array}{l}\text { - Utilized super capacitive energy storage in MG to feed fault cur- } \\
\text { rents in the situation of communication failure } \\
\\
\text { communication is attacked or encountered a failure }\end{array}$ \\
\hline [26] & 43 & 13 & Centralized & $\begin{array}{l}\text { - Identified the topology utilizing Prim-aided Dijkstra algorithm to } \\
\text { adapt the settings according to the shortest route path } \\
\checkmark \text { Cleared the fault by removing the minimum protection zone } \\
\text { Ensured the continuity of supplying the load }\end{array}$ \\
\hline$[27]$ & 37 & 14 & Centralized & $\begin{array}{l}\text { - Detected fault based on measured fault current and pre-set } \\
\text { threshold by modified cumulative sum average approach } \\
\\
\text { Fast detection of the faults in MGs }\end{array}$ \\
\hline$[28]$ & 37 & 15 & Decentralized & $\begin{array}{l}\text { - Used a protection scheme based on high impedance differential } \\
\text { relay in FDERS to reduce the maloperation of protection devices } \\
\text { due to the variation of current } \\
\text { Enhanced controllability } \\
\text { Improved system robustness }\end{array}$ \\
\hline [29] & 32 & 16 & Centralized & $\begin{array}{l}\text { - Utilized a central control and monitoring infrastructure for } \\
\text { adaptive OCRs' settings for } \mathrm{MG}^{\prime} \text { protection } \\
\\
\text { gation }\end{array}$ \\
\hline$[30]$ & 31 & 17 & Centralized & $\begin{array}{l}\text { - Utilized the dynamic state estimation of the system's parameters as } \\
\text { phasor data to estimate the MG's status } \\
\text { Obtained more data accuracy and computational speed in } \\
\text { comparison to the conventional centralized approaches for MG's } \\
\text { protection }\end{array}$ \\
\hline$[31]$ & 30 & 18 & Centralized & $\begin{array}{l}\text { - Notified each CB's or DG's status by sending messages from the } \\
\text { OCRs to the MCPU and adapt the OCRs' settings by sending back } \\
\text { the message from MCPU } \\
\\
\text { Supported protection of MG in the critical time } \\
\text { with the provided fixed delay by applying the WiMAX network along } \\
\text { waptive scheme }\end{array}$ \\
\hline$[32]$ & 29 & 19 & Decentralized & $\begin{array}{l}\text { - Applied the fuse relay along with logic gate for adaptive over- } \\
\text { current protection to protect lines and feeders } \\
\text { Improved selectivity, reliability, and speed of protection utiliz- } \\
\text { ing fuse relay and logic gate }\end{array}$ \\
\hline$[33]$ & 29 & 20 & Centralized & $\begin{array}{l}\text { - Utilized the Pearson correlation coefficients of MG's elements for } \\
\text { detection of topological changes and ANN-SVM for detection of } \\
\text { MG's status } \\
\swarrow \text { Ensured the reliability of the MG's operation }\end{array}$ \\
\hline$[34]$ & 29 & 21 & Centralized & $\begin{array}{l}\text { - Utilized } \mu \text { PMUs to obtain Thevenin impedance for monitoring the } \\
\text { MG and adapt the OCRs' settings due to line or source outage } \\
\text { contingencies } \\
\\
\text { No need to relay-to-relay communications }\end{array}$ \\
\hline$[35]$ & 29 & 22 & Decentralized & $\begin{array}{l}\text { - Applied a fault detection procedure based on the WPT } \\
- \text { Insensitive to the MG mode of operation }\end{array}$ \\
\hline$[36]$ & 29 & 23 & Centralized & $\begin{array}{l}\text { - Utilized an IDM with an adaptive control strategy to enhance the } \\
\text { system operation during the islanded mode } \\
\text { Is not affected by the changes in load or generation } \\
\text { Remained the DG units stable during the transition period by } \\
\text { decreases the overshoot, undershoot, and settling times of the DG } \\
\text { parameters } \\
\text { Improve the power quality due to the ability to detect low } \\
\text { strength signals results in smaller signal distortion by the DGs }\end{array}$ \\
\hline
\end{tabular}


Table 1. Continued

\begin{tabular}{|c|c|c|c|c|}
\hline Reference & Citations & Rank & $\begin{array}{l}\text { Category (Centralized or } \\
\text { decentralized) }\end{array}$ & Remarks/advantages \\
\hline$[37]$ & 27 & 24 & Decentralized & $\begin{array}{l}\text { Determined the operation mode by comparing the angle of im- } \\
\text { pedance's zero-sequence obtained by the local information and } \\
\text { identified the fault through zero-sequence of fault current } \\
\\
\text { operationg capable to adjust the protection strategies in the different } \\
\text { Detected the faults and clear the faulted zone without the } \\
\text { communication }\end{array}$ \\
\hline$[38]$ & 25 & 25 & Centralized & $\begin{array}{l}\text { - Utilized central control and monitoring infrastructure to adapt the } \\
\text { settings to OCRs in MG applying FPGA for decision-making } \\
\text { Reduce fault detection time by applying FPGA }\end{array}$ \\
\hline [39] & 24 & 26 & $\#$ & $\begin{array}{l}\text { - Reviewed studies associated with adaptive protection considering } \\
\text { DGs }\end{array}$ \\
\hline$[40]$ & 24 & 27 & Centralized & $\begin{array}{l}\text { - Trained two estimation-based ANN models in control center with } \\
\text { real-time measurements to detect the fault on the line and its } \\
\text { location } \\
\text { Enhanced the reliable operation for the different operational } \\
\text { modes } \\
\text { intelligently }\end{array}$ \\
\hline$[41]$ & 24 & 28 & Centralized & $\begin{array}{l}\text { - Presented an adaptive control strategy for MG } \\
\text { limproved the existing controllers by obtaining a response with } \\
\text { occurrence }\end{array}$ \\
\hline$[42]$ & 22 & 29 & Centralized & $\begin{array}{l}\text { - Utilized an IDM for a MG with multi PVs with the harmonic } \\
\text { amplification factor of the PCC's voltage as an islanding detection } \\
\text { parameter } \\
\text { Improved stability of MG }\end{array}$ \\
\hline$[43]$ & 22 & 30 & Centralized & $\begin{array}{l}\text { - Utilized a three-stage fast detection model of dynamic security of } \\
\text { status in MG for protection purposes } \\
\\
\text { Fast and accurate to prevent any unnecessary tripping of DERs }\end{array}$ \\
\hline
\end{tabular}

\#: Review paper

Reference [14] proposes a protection strategy that is effective for both modes of operations in the MG. The proposed strategy is based on microprocessor-based OCRs for MGs that makes it independent of communication infrastructures.

Adaptive relaying and non-adaptive relaying schemes are discussed in [15], with a concentration on a suitable protection scheme conserving dynamic fault currents and changing the network topologies.

In [16] a protection strategy is proposed that is based on the communication-assisted OCRs to provide adaptive settings according to their operation and respond to the different fault current levels. This is done by the synchronous generator in the MG to provide sufficient fault current for OCRs.

In [17] a centralized adaptive protection scheme is proposed that focused on the selection of alternative settings for OCRs utilizing programmed logic and utilized IEC 61850 as communication infrastructure. The proposed adaptive protection scheme is currently being installed at Hailuoto Island in Finland.
In [18] an adaptive protection scheme for MGs is proposed that is suitable for various fault conditions independent of the operating mode. This is performed by monitoring the MG and updating the fault current in OCRs according to the variations (i.e., faults). The proposed adaptive protection scheme also utilizes auto reclosers, which enable the proposed scheme to recover faster from the fault and consequently improves the stability of the MG.

Reference [19] briefly reviews the protection issues in AC MGs along with focusing on overcoming the limitations of protection schemes and improvement of reliability in communication infrastructures for adaptive protection schemes by providing alternate techniques in the case of their failures.

In [20] a summary of works was carried out in the scope of MG protection. This paper analyzed the MG's protection methods by concentration on issues such as changing the OCRs' settings, the connection status of DGs including the different types of DGs, the application of fault current limiters (FCLs), and finally the adaptive protection. 
The authors in [21] proposed an adaptive protection scheme for MGs utilizing energy storage devices (ESDs) to increase the fault current magnitude in a cyber-attack or communication failures in the islanded mode.

Reference [22] presented a hybrid MG's protection system consisting of a traditional differential protection scheme with low communications load along with an adaptive scheme to increase the accuracy and precision of the whole protection scheme and reducing the overall communications cost. To this end, the adaptive protection scheme is implemented as the default scheme, while differential protection is utilized in the situation of the high-risk protection zone.

Reference [23] proposed an adaptive directional OCR technique based on the positive-sequence (PSQ) and negative-sequence (NSQ) currents in MGs. In this technique, the fault currents (before the occurrence of the fault and during the fault situation) are obtained and in a comparison-based procedure the fault is detected and finally, the settings corresponding to the OCRs are adapted.

Similarly in [24], the fault is detected according to the fault current characteristic (i.e., the negative sequence of fault current is used to detect the line-to-line faults and the zero sequence is used to detect single line-to-ground fault) in inverter-based DGs. The fault current components are obtained utilizing the Fourier filter algorithm. Moreover, the proposed adaptive algorithm used the voltage and fault current components to calculate the system impedance in an online manner. Then, the method adaptively changes the setting value of OCRs according to the operation mode by comparing with the system impedance and MG side impedance.

In [25] the authors utilized super capacitive energy storage to feed fault currents and prevent the control system from being collapsed on the communication failure.

In [26], a technique is presented to protect MG adaptively by continuous monitoring. The technique utilized the Prim-aided Dijkstra algorithm to identify the shortest route from the faulted point to the nearest source in an online manner and determine the topology. Then the settings of OCRs have varied adaptively according to the priority of the identified shortest path.

Reference [27] proposed a differential current-based method based on measured current and pre-set threshold to detect, classify and locate the faults by modified cumulative sum average approach technique.

In [28] an adaptive protection scheme is performed for MG that is based on high impedance differential relays in flexible distribution of energy and storage resources (FDERS). The proposed scheme reduces the maloperation of protection devices due to the variation of the current waveform in FDERS.

Reference [29] utilized central control and monitoring unit for adaptive settings of MG. Implementation of the proposed scheme leads to fault mitigation and ensures reliability in the protection system.

Dynamic state estimation is used in [30] as an adaptive protection scheme for MGs. In this study, the estimated state variables corresponding to each distributed energy resource (DER) in MG are converted to phasor data and collected to the DER management system (DERMS) to be used as the input of the system for the state estimation of MG.

In [31] an adaptive protection scheme for MG is proposed that utilized the bidirectional communication capability based on worldwide interoperability for microwave access (WiMAX). In this scheme, each OCR sends a message including the status of components (i.e., CBs and DGs) to the MG central protection unit (MCPU) as soon as any change occurs. On the other hand, an update status message is sent to the OCRs to notify the new settings.

Reference [32] proposed an adaptive overcurrent protection based on fuses and OCRs in a MG to protect lines and feeders. In this scheme, the settings of OCRs are adapted by logic gates of CBs related to the OCRs.

In [33] the Pearson correlation coefficients of elements of MG are obtained from a data mining procedure and the state of MG is detected through a hybrid artificial neural network and support vector machine (ANN-SVM). Based on the detection algorithm, the OCRs' settings can be modified according to any reconfigurations.

In [34] an adaptive protection scheme is proposed based on online detection of uncertainties (i.e., line and power plant outages) by monitoring of Thevenin impedance estimation that is obtained by micro phasor measurement units ( $\mu$ PMUs) installed between transmission network and the PCC of MG. The OCRs are updated according to the phasor data that are gathered from the network.

An adaptive protection technique is presented in [35] in which a fault detection procedure is applied that utilized the wavelet packet transform (WPT). The first set of WPT coefficients is utilized to detect the fault and the second set is used to locate it.

In [36] a new hybrid island detection method (IDM) with an adaptive control strategy is proposed to enhance the system operation during the islanded mode. The IDM firstly detects the islanded mode in a distribution system, and then an adaptive control strategy is utilized to ensure stable operation of islanded mode. This is done utilizing error rates of system parameters such as voltage and power to readjust the generator controllers and maintain the system's stability.

An adaptive protection scheme for MGs in which the fault in both MG's operation modes is detected through the structures of the zero-sequence of fault current is proposed in [37]. Also, the OCR can discriminate the operation mode by comparing the angle of zero sequence impedance obtained by the local information.

In [38] the authors proposed a method for MG's protection that is suitable for both modes of operation of MG against all different types of faults. In the proposed method, a central control and monitoring unit is utilized for adaptive OCRs' settings by detecting multifunction of OCRs. The decision-making procedure for adaptation of the settings is applied through field programmable gate array (FPGA).

Reference [39] presented a comprehensive survey on MG's protection with emphasis on adaptive protection considering DGs. Also, the newly developed schemes concerning adaptive protection of MGs and distribution systems with DGs are presented. 
An adaptive overcurrent protection strategy for MG according to an estimation model utilizing two ANNs is proposed in [40]. The first ANN model is trained with online measurements to detect the fault on the line and the second ANN is used for fault allocation. In the proposed scheme, the settings of OCRs are obtained by a linear programming problem in the central system and adapted to OCRs according to the detection procedure.

In [41], an adaptive control strategy is proposed to improve the performance of the existing controllers and ensure reliable operation of resources in MGs. The proposed controller monitors the response of a controlled device and modulates its control setpoint to obtain a response with limited over or undershoot.

In [42] an IDM is presented for a MG with multi PVs. In the proposed scheme island detection is performed as an adaptive procedure according to the status of PV systems. Also, the islanding detection parameter is the harmonic amplification factor of the PCC's voltage.

A three-stage adaptive model for the detection of the dynamic security status of MG is presented in [43]. In the first stage, the offline analysis of DERs for designing dynamic security models in MG is performed and the settings of OCRs are determined. In the second stage, the obtained calculations from the first stage model are used to determine the protection zones of DERs in an online manner. And finally, in the third stage, the online detection of dynamic security status of MG is performed and protects the MG by issuing the proper protection trip commands.

\section{CONCLUSIONS}

This paper presented a comprehensive statistics-based review on MG's protection with a concentration on adaptive protection. The observations of the investigated publications of MG illustrate that protection issues include a significant part of MG's topics. Moreover, it can be concluded that most publications on adaptive protection of MGs are based on centralized infrastructures to issue adaptive actions that is due to the significant development of telecommunication and communication systems in the power system. It is hoped that this work will be useful to the researchers in the field of MG's protection and especially the adaptive protection of MG to investigate the concentrations of researches for future studies.

\section{REFERENCES}

[1] Y. Yoldaş, A. Önen, S. M. Muyeen, A. V. Vasilakos, and İ. Alan, "Enhancing smart grid with microgrids: Challenges and opportunities," Renew. Sust. Energ. Rev., vol. 72, pp. 205-14, 2017, https://doi.org/10.1016/j.rser.2017.01.064.

[2] M. Monadi, C. Gavriluta, A. Luna, J. I. Candela, and P. Rodriguez, "Centralized protection strategy for medium voltage DC microgrids," IEEE Trans. Power Deliv., vol. 32, no. 1, pp. 430-40, 2016, https://doi.org/10.1109/TPWRD.2016.2600278.
[3] A. Hasankhani and S. M. Hakimi, "Stochastic energy management of smart microgrid with intermittent renewable energy resources in electricity market," Energy., vol. 219, pp. 119668-82, 2021, https://doi.org/10.1016/j.energy.2020.119668.

[4] A. Rezaee Jordehi, “An improved particle swarm optimisation for unit commitment in microgrids with battery energy storage systems considering battery degradation and uncertainties," Int. J. Energ. Res., vol. 45, no. 1, pp. 727-44, 2021, https://doi.org/10. 1002/er.5867.

[5] S. Chandak and P. K. Rout, "The implementation framework of a microgrid: A review," Int. J. Energ. Res., vol. 45, no. 3, pp. 3523-47, 2021, https://doi.org/10.1002/er.6064.

[6] Y. C. Tsao, V. V. Thanh, and Q. Wu, "Sustainable microgrid design considering blockchain technology for real-time pricebased demand response programs," Int. J. Electr. Power Energ. Syst., vol. 125, pp. 106418-31, 2021, https://doi.org/10.1016/j. ijepes.2020.106418.

[7] C. Wang, Z. Zhang, O. Abedinia, and S. G. Farkoush, "Modeling and analysis of a microgrid considering the uncertainty in renewable energy resources, energy storage systems and demand management in electrical retail market," J. Energ. Stor., vol. 33, pp. 102111-23, 2021, https://doi.org/10.1016/j.est.2020.102111.

[8] D. Gutierrez-Rojas, P. H. Nardelli, G. Mendes, and P. Popovski, "Review of the state of the art on adaptive protection for microgrids based on communications," IEEE Trans. Industr. Inform., vol. 17, no. 3, pp. 1539-52, 2020, https://doi.org/10.1109/TII.2020.3006845.

[9] A. Cagnano, E. De Tuglie, and P. Mancarella, "Microgrids: Overview and guidelines for practical implementations and operation," Appl. Energ., vol. 258, pp. 114039-56, 2020, https:// doi.org/10.1016/j.apenergy.2019.114039.

[10] K. Wu, Q. Li, Z. Chen, J. Lin, Y. Yi, and M. Chen, "Distributed optimization method with weighted gradients for economic dispatch problem of multi-microgrid systems," Energy, vol. 222, pp. 119898-908, 2021, https://doi.org/10.1016/j.energy.2021. 119898.

[11] A. Sharma and B. K. Panigrahi, "Phase fault protection scheme for reliable operation of microgrids," IEEE Trans. Ind. Appl., vol. 54, no. 3, pp. 2646-55, 2017, https://doi.org/10.1109/TIA.2017. 2787691.

[12] A. K. Tiwari, S. R. Mohanty, and R. K. Singh, "Review on protection issues with penetration of distributed generation in distribution system," in 2014 Int. Electr. Eng. Congr. (iEECON), pp. 1-4, 2014

[13] S. Mirsaeidi, X. Dong, and D. M. Said, "Towards hybrid AC/DC microgrids: Critical analysis and classification of protection strategies," Renew. Sust. Energ. Rev., vol. 90, pp. 97-103, 2018, https:// doi.org/10.1016/j.rser.2018.03.046.

[14] M. A. Zamani, T. S. Sidhu, and A. Yazdani, "A protection strategy and microprocessor-based relay for low-voltage microgrids," IEEE Trans. Power Deliv., vol. 26, no. 3, pp. 1873-83, 2011, https://doi. org/10.1109/TPWRD.2011.2120628.

[15] V. Telukunta, J. Pradhan, A. Agrawal, M. Singh, and S. G. Srivani, "Protection challenges under bulk penetration of renewable energy resources in power systems: A review," CSEE J. Power Energ. Syst., vol. 3, no. 4, pp. 365-79, 2017, https://doi.org/10.17775/ CSEEJPES.2017.00030.

[16] L. Che, M. E. Khodayar, and M. Shahidehpour, "Adaptive protection system for microgrids: Protection practices of a functional 
microgrid system," IEEE Electrif. Mag., vol. 2, no. 1, pp. 66-80, 2014, https://doi.org/10.1109/MELE.2013.2297031.

[17] H. Laaksonen, D. Ishchenko, and A. Oudalov, "Adaptive protection and microgrid control design for Hailuoto Island," IEEE Trans. Smart Grid, vol. 5, no. 3, pp. 1486-93, 2014, https://doi.org/ 10.1109/TSG.2013.2287672.

[18] R. Sitharthan, M. Geethanjali, and T. K. Pandy, "Adaptive protection scheme for smart microgrid with electronically coupled distributed generations," Alex. Eng. J., vol. 55, no. 3, pp. 2539-50, 2016, https://doi.org/10.1016/j.aej.2016.06.025.

[19] A. A. Memon and K. Kauhaniemi, "A critical review of AC microgrid protection issues and available solutions," Electr. Power Syst. Res., vol. 129, pp. 23-31, 2015, https://doi.org/10.1016/j.epsr. 2015.07.006.

[20] S. A. Hosseini, H. A. Abyaneh, S. H. Sadeghi, F. Razavi, and A. Nasiri, "An overview of microgrid protection methods and the factors involved," Renew. Sust. Energ. Rev., vol. 64, pp. 174-86, 2016, https://doi.org/10.1016/j.rser.2016.05.089.

[21] H. F. Habib, C. R. Lashway, and O. A. Mohammed, "A review of communication failure impacts on adaptive microgrid protection schemes and the use of energy storage as a contingency," IEEE Trans. Ind. Appl., vol. 54, no. 2, pp. 1194-207, 2017, https://doi. org/10.1109/TIA.2017.2776858.

[22] T. S. Ustun and R. H. Khan, "Multiterminal hybrid protection of microgrids over wireless communications network," IEEE Trans. Smart Grid, vol. 6, no. 5, pp. 2493-500, 2015, https://doi.org/10. 1109/TSG.2015.2406886.

[23] H. Muda and P. Jena, "Superimposed adaptive sequence current based microgrid protection: a new technique," IEEE Trans. Power Deliv., vol. 32, no. 2, pp. 757-67, 2016, https://doi.org/10.1109/ TPWRD.2016.2601921.

[24] Y. Han, X. Hu, and D. Zhang, "Study of adaptive fault current algorithm for microgrid dominated by inverter based distributed generators," in The 2nd Int. Symp. Power Electron. Distrib. Gener. Syst., pp. 852-4, 2010.

[25] H. F. Habib, A. A. Mohamed, M. El Hariri, and O. A. Mohammed, "Utilizing supercapacitors for resiliency enhancements and adaptive microgrid protection against communication failures," Electr. Power Syst. Res., vol. 145, pp. 223-33, 2017, https://doi.org/ 10.1016/j.epsr.2016.12.027.

[26] O. G. Swathika and S. Hemamalini, "Prims-aided Dijkstra algorithm for adaptive protection in microgrids," IEEE J. Emerg. Sel. Topics Power Electron., vol. 4, no. 4, pp. 1279-86, 2016, https://doi. org/10.1109/JESTPE.2016.2581986.

[27] S. Dhar and P. K. Dash, "Differential current-based fault protection with adaptive threshold for multiple PV-based DC microgrid," IET Renew. Power Gener., vol. 11, no. 6, pp. 778-90, 2017, https://doi.org/10.1049/iet-rpg.2016.0577.

[28] M. A. Haj-ahmed and M. S. Illindala, "Investigation of protection schemes for flexible distribution of energy and storage resources in an industrial microgrid," in 2014 IEEE/IAS $50^{\text {th }}$ Ind. Commer. Power Syst. Tech. Conf., pp. 1-10, 2014.

[29] M. R. Islam and H. A. Gabbar, "Study of micro grid safety \& protection strategies with control system infrastructures," Smart
Grid Renew. Energ., vol. 3, no. 1, pp. 1-9, 2012, https://doi.org/10. 4236/sgre.2012.31001.

[30] S. Choi and A. S. Meliopoulos, "Effective real-time operation and protection scheme of microgrids using distributed dynamic state estimation," IEEE Trans. Power Deliv., vol. 32, no. 1, pp. 504-14, 2016, https://doi.org/10.1109/TPWRD.2016.2580638.

[31] T. S. Ustun, R. H. Khan, A. Hadbah, and A. Kalam, "An adaptive microgrid protection scheme based on a wide-area smart grid communications network," in 2013 IEEE Latin-America Conf. Commun., pp. 1-5, 2013.

[32] E. C. Piesciorovsky and N. N. Schulz, "Fuse relay adaptive overcurrent protection scheme for microgrid with distributed generators," IET Gener., Transm. Distrib., vol. 11, no. 2, pp. 540-9, 2017, https://doi.org/10.1049/iet-gtd.2016.1144.

[33] H. Lin, K. Sun, Z. H. Tan, C. Liu, J. M. Guerrero, and J. C. Vasquez, "Adaptive protection combined with machine learning for microgrids," IET Gener., Transm. Distrib., vol. 13, no. 6, pp. 770-9, 2019, https://doi.org/10.1049/iet-gtd.2018.6230.

[34] M. G. Zanjani, K. Mazlumi, and I. Kamwa, "Application of $\mu$ PMUs for adaptive protection of overcurrent relays in microgrids," IET Gener., Transm. Distrib., vol. 12, no. 18, pp. 4061-8, 2018, https://doi.org/10.1049/iet-gtd.2018.5898.

[35] S. A. Saleh, "Signature-coordinated digital multirelay protection for microgrid systems," IEEE Trans. Power Electron., vol. 29, no. 9, pp. 4614-23, 2013, https://doi.org/10.1109/TPEL.2013.2285978.

[36] A. Pouryekta, V. K. Ramachandaramurthy, N. Mithulananthan, and A. Arulampalam, "Islanding detection and enhancement of microgrid performance," IEEE Syst. J., vol. 12, no. 4, pp. 3131-41, 2017, https://doi.org/10.1109/JSYST.2017.2705738.

[37] K. Dang, X. He, D. Bi, and C. Feng, "An adaptive protection method for the inverter dominated microgrid," in 2011 Int. Conf. Electr. Mach. Syst., pp. 1-5, 2011.

[38] M. R. Islam and H. A. Gabbar, "Analysis of microgrid protection strategies," in 2012 Int. Conf. Smart Grid (SGE), pp. 1-6, 2012.

[39] P. H. Barra, D. V. Coury, and R. A. Fernandes, "A survey on adaptive protection of microgrids and distribution systems with distributed generators," Renew. Sust. Energ. Rev., vol. 118, pp. 109524-39, 2020, https://doi.org/10.1016/j.rser.2019.109524.

[40] H. Lin, J. M. Guerrero, C. Jia, Z. H. Tan, J. C. Vasquez, and C. Liu, "Adaptive overcurrent protection for microgrids in extensive distribution systems," in IECON 2016-42nd Annu. Conf. IEEE Ind. Electron. Soc., pp. 4042-7, 2016.

[41] A. Mehrizi-Sani and R. Iravani, "Online set point modulation to enhance microgrid dynamic response: Theoretical foundation," IEEE Trans. Power Syst., vol. 4, no. 27, pp. 2167-74, 2012, https:// doi.org/10.1109/TPWRS.2012.2190532.

[42] S. Dhar and P. K. Dash, "Adaptive threshold based new active islanding protection scheme for multiple PV based microgrid application," IET Gener., Transm. Distrib., vol. 11, no. 1, pp. 118-32, 2017, https://doi.org/10.1049/iet-gtd.2016.0679.

[43] S. Teimourzadeh, F. Aminifar, M. Davarpanah, and M. Shahidehpour, "Adaptive protection for preserving microgrid security," IEEE Trans. Smart Grid, vol. 10, no. 1, pp. 592-600, 2017, https:// doi.org/10.1109/TSG.2017.2749301. 